\title{
Anti-irritant and anti-inflammatory effects of glycerol and xylitol in sodium lauryl sulfate-induced acute irritation
}

Effects of polyols in irritant contact dermatitis

E Szél ${ }^{1}$, H Polyánka ${ }^{2}, \mathrm{~K}$ Szabó ${ }^{2}, \mathrm{P}$ Hartmann $^{3}$, D Degovics ${ }^{1}$, B Balázs ${ }^{4}$, IB Németh ${ }^{1}, \mathrm{C}$ Korponyai $^{1}$, E Csányi ${ }^{4}$, J Kaszaki $^{3}$, S Dikstein ${ }^{5}$, K Nagy $^{6}$, L Kemény ${ }^{1,2}$, G Erős ${ }^{1,7}$

${ }^{1}$ Department of Dermatology and Allergology, University of Szeged

${ }^{2}$ MTA SZTE Dermatological Research Group

${ }^{3}$ Institute of Surgical Research, University of Szeged

${ }^{4}$ Department of Pharmaceutical Technology, University of Szeged

${ }^{5}$ Unit of Cell Pharmacology, Hebrew University

${ }^{6}$ Department of Oral Surgery, University of Szeged

${ }^{7}$ Department of Oral Biology and Experimental Dental Research, University of Szeged

Text word count: 2915

Number of tables: 2

Number of figures: 9

\section{Corresponding author:}

Edit Szél, M.D.

Department of Dermatology and Allergology, Faculty of Medicine, University of Szeged,

Korányi fasor 6., H-6720, Szeged, Hungary

Tel: +36-70-776-1104

Fax: +36-62-545-954

E-mail: szeledit@hotmail.com 
Authors have no conflicts of interest to declare. 


\section{Abstract}

BACKGROUND: Glycerol is known to possess anti-irritant and hydrating properties and previous studies suggested that xylitol may also have similar effects.

OBJECTIVE: Our aim was to study whether different concentrations of these polyols restore skin barrier function and soothe inflammation in sodium lauryl sulfate (SLS)-induced acute irritation.

METHODS: The experiments were performed on male SKH-1 hairless mice. The skin of the dorsal region was exposed to SLS (5\%) for $3 \mathrm{~h}$ alone or together with $5 \%$ or $10 \%$ of glycerol, respectively. Further 2 groups received xylitol solutions (8.26\% and $16.52 \%$, respectively) using the same osmolarities, which were equivalent to those of the glycerol treatments. The control group was treated with purified water. Transepidermal water loss (TEWL) and skin hydration were determined. Microcirculatory parameters of inflammation were observed by means of intravital videomicroscopy (IVM). Furthermore, accumulation of neutrophil granulocytes and lymphocytes, the expression of inflammatory cytokines and SLS penetration were assessed, as well.

RESULTS: Treatment with the $10 \%$ of glycerol and both concentrations of xylitol inhibited the SLS-induced elevation of TEWL, moderated the irritant-induced increase in dermal blood flow and in the number of leukocyte-endothelial interactions. All concentrations of the applied polyols improved hydration and prevented the accumulation of lymphocytes near the treatment site. At the mRNA level, neither glycerol nor xylitol influenced the expression of interleukin-1 alpha. However, expression of interleukin-1 beta was significantly decreased by the $10 \%$ glycerol treatment, while expression of tumor necrosis factor-alpha decreased upon the same treatment, as well as in response to xylitol. Higher polyol treatments decreased the SLS penetration to the deeper layers of the stratum corneum. 
CONCLUSION: Both of the analyzed polyols exert considerable anti-irritant and antiinflammatory properties, but the effective concentration of xylitol is lower than that of glycerol.

Keywords: glycerol; xylitol; sodium lauryl sulfate, acute irritation 


\section{Introduction}

Irritant contact dermatitis (ICD) is a non-immunologic and non-specific inflammatory disorder of the skin that is caused by physical, mechanical or chemical challenges[1]. Irritation is accompanied by disruption of the barrier and dehydration of the uppermost layer of the skin. Hence, agents contributing to skin hydration and the maintenance of its homeostasis are required for treatment and prevention of ICD. Glycerol, applied in several external formulations, meets these requirements. This polyol exerts anti-irritant effect and acts as a humectant[2, 3]. Moreover, glycerol improves barrier function, hydrates the skin, stabilizes skin collagen and accelerates wound healing $[4,5]$. All these raise the question whether structurally similar polyol molecules exhibit similar properties. Xylitol is a naturally occurring polyol, which is a widely used substitute of sugar. Recent studies indicated that xylitol can be utilized as humectant and moisturizer, as well[6, 7]. In our previous study we have shown that both glycerol and xylitol suppresses the $0.1 \%$ sodium lauryl sulfate (SLS)induced acute skin irritation[8]. However, it is not clear whether these polyols are able to provide protection against a more serious irritation. Despite their similar chemical structure, in vitro examinations suggest that glycerol and xylitol evoke different gene expression changes in keratinocytes. Glycerol was found to decrease the expression of human leukocyte antigen DR while xylitol increased filaggrin expression[9]. Currently, we have a lot of data on the effects of glycerol on the skin and its potential mechanism of action[5], but much less information is available on the applicability of xylitol.

Our goal was to examine and compare the effects of different concentrations of glycerol and xylitol on skin barrier function, skin hydration, dermal microcirculation, increase our knowledge on the cellular and molecular factors of inflammation and SLS penetration using

an animal

model

of

skin

irritation. 


\section{Materials and Methods}

Animals

12-15-week-old male SKH-1 hairless mice were housed in plastic cages in a thermoneutral environment with a $12 \mathrm{~h}$ light-dark cycle and had access to standard laboratory chow and water ad libitum. All interventions were in full accordance with the NIH guidelines and protocols were approved by the Ethical Committee for the Protection of Animals in Scientific Research at the University of Szeged (license number: V./145/2013). Animals were anesthetized with a mixture of ketamine $(90 \mathrm{mg} / \mathrm{kg}$ body weight) and xylazine $(25 \mathrm{mg} / \mathrm{kg}$ body weight) administered intraperitoneally. In the end of the experiments, mice were euthanized with an overdose of ketamine $(300 \mathrm{mg} / \mathrm{kg})$.

\section{Experimental design (Fig. 1)}

Mice were randomly allocated into six groups. Group $1(n=23)$ served as a control treated with purified water. Group $2(n=23)$ was exposed to a 5\% solution of SLS. In group $3(n=23)$, the solution applied to the skin contained SLS (5\%) and glycerol of 5\%. The animals of group $4(n=23)$ received a solution with SLS $(5 \%)$ and xylitol of $8.26 \%$. Group $5(n=23)$ was treated with SLS (5\%) and glycerol of $10 \%$, while in group $6(n=23)$, SLS (5\%) and xylitol of $16.52 \%$ were applied to the skin. SLS and the polyols were dissolved in purified water. Osmolarities of appropriate glycerol and xylitol solutions were equivalent. Groups and treatments are summarized in Table 1. In each group, 15 mice were randomly chosen for patch testing followed by attenuated total reflectance Fourier transformed infrared spectroscopy (ATR-FTIR) or determination of transepidermal water loss (TEWL) and skin hydration. After sacrificing the animals, the treated skin was excised and divided into two parts. One part was used to determine either myeloperoxidase (MPO) activity or the expression of inflammatory cytokines (interleukin-1 alpha and beta - IL- $1 \alpha$ and IL-1ß, tumor 
necrosis factor-alpha - TNF- $\alpha$ ) by means of real time polymerase chain reaction (RT-PCR). Half of the remaining part was fixed in a solution of formaldehyde for routine histology and the other half was subjected to immunofluorescent staining.

Dorsal skin fold chamber was implanted to the dorsal region of the other 8 mice in order to study the microcirculation.

\section{Patch testing}

In closed patch tests, extra-large Finn Chambers (diameter of $18 \mathrm{~mm}$ ) and corresponding filter discs soaked with $120 \mu \mathrm{L}$ of the test solutions were applied to the dorsal region for 3 hours using Scanpore tape. The relative humidity was $40-50 \%$ and the ambient temperature was kept at $20-22{ }^{\circ} \mathrm{C}$.

Combined tape stripping and ATR-FTIR experiments

For the ATR-FTIR experiments, corneocytes were obtained with adhesive cellophane tape from the patch-tested mice dorsal region. This was repeated up to 25 strips recording an IR spectrum after each third tape strip. Every first tape with one strip was discarded because of the possibility of surface contamination. The untreated dorsal skin of mice was stripped and measured with the same method. ATR-FTIR spectra were recorded with an Avatar 330 FT-IR spectrometer equipped with a horizontal ATR crystal $\left(\mathrm{ZnSe}, 45^{\circ}\right)$, between 4000 and 400 $\mathrm{cm}^{-1}$, at an optical resolution of $4 \mathrm{~cm}^{-1}$. 64 scans were co-added and all spectral manipulations were performed by using Thermo Scientific's GRAMS/AI Suite software. In order to obtain a reference spectrum of the API, a KBr pellet containing $0.5 \mathrm{mg}$ SLS was prepared and used. The spectra of the preparations were measured. The spectra of treated and untreated samples were also recorded. Each spectrum of the individual layers of the treated stratum corneum (SC) - containing three strips - was corrected with the spectrum of the own untreated layer. To ensure that no absorbances from the skin itself are remaining and 
interfering the results, spectra of untreated control skin samples were also subtracted from spectra of water treated control skin samples. No ATR correction was performed.

Measurement of TEWL and skin hydration

TEWL and hydration measurements were carried out before application of Finn Chambers and 15 min after their removal. TEWL was assessed with a Tewameter TM300 and skin hydration was determined with a Corneometer CM825.

Implantation of dorsal skin fold chamber

Eight mice of each group were implanted by dorsal skin fold chambers according to a previously described method[10]. After a 24-h recovery period, a filter disc with $120 \mu \mathrm{L}$ of the test solution was applied to the non-wounded side and covered with a non-permeable film for $3 h$.

Intravital videomicroscopy

The microcirculation was visualized with a fluorescent intravital videomicroscope (Zeiss). The anesthetized mice received a retrobulbar injection of $2 \%$ fluorescein isothiocyanatelabeled dextran and $0.2 \%$ rhodamine- $6 \mathrm{G}$ in order to visualize microcirculation and to stain leukocytes. During examinations, the tissue was superfused with $37^{\circ} \mathrm{C}$ saline. The microcirculatory parameters were assessed quantitatively off-line by frame-to-frame analysis of the videotaped images. The red blood cell velocity $(\mathrm{RBCV}, \mu \mathrm{m} / \mathrm{s})$ was measured in 4 separate fields of view, in at least 6 capillaries. Leukocyte-endothelial cell interactions were analyzed within 5 postcapillary venules per animal. Adherent leukocytes were defined in each vessel segment as cells that did not move or detach from the endothelial lining within an observation period of 30 s.

Routine histology 
The hematoxylin-eosin stained $3 \mu \mathrm{m}$ thick coded sections were analyzed with Pannoramic Viewer software. Thickness of viable epidermis (stratum corneum not included) was measured at 20 different points of each section.

Immunofluorescent staining for lymphocytes

The $6 \mu \mathrm{m}$ cryosections were stained with phycoerythrine conjugated rat-anti mouse CD3 antibody in 1:20 dilution. Nuclear staining with 4', 6-diamidino-2-phenylindole (DAPI, 1:100 dilution, 10 min incubation) was also performed. The sections were examined with an AxioImager.Z1 microscope (Zeiss). Images of the sections were analyzed with ImageJ software. The number of CD3+ cells was referred to 50 basal keratinocytes.

\section{RT-PCR}

Total RNA was isolated using RNeasy Mini Kit (Qiagen) according to the manufacturer's instructions. For PCR, amplification was carried out in duplicates of a total volume of $20 \mu \mathrm{L}$ per sample. Subsequent RT-PCR was performed to quantify transcript abundance using custom primer sets and the Universal Probe Library (Roche) with an iQ Supermix (Bio-Rad) in an LC480 real-time PCR machine (Roche). Relative quantification was carried out using the $2^{-\Delta \Delta \mathrm{CT}}$ method and $\beta 2$-microglobuline as an endogenous control.

\section{Tissue MPO activity}

The activity of MPO, a marker of polymorphonuclear granulocyte accumulation in inflamed tissues, was measured by the method of Kuebler et al.[11]. The MPO activities of the samples were measured at $450 \mathrm{~nm}(\mathrm{UV}-1601$ spectrophotometer) and the data were referred to the protein content.

Statistical analysis 
Data analysis was performed with SigmaStat for Windows software. Differences among groups were analyzed with Kruskal-Wallis one-way analysis of variance on ranks, followed by Dunn method for pairwise multiple comparison. $\mathrm{P}<0.05$ was considered statistically significant. 


\section{Results}

TEWL displayed only slight changes in the purified water-treated control group, while exposure to SLS led to a significant elevation in TEWL and application of glycerol of 5\% failed to prevent this alteration. Xylitol and glycerol at concentrations of $8.26 \%$ and $10 \%$, respectively, improved barrier function. Xylitol of $16.52 \%$ restored TEWL values to the control levels after irritant treatment (Fig. 2).

In skin hydration, neither purified water in the control group nor SLS alone resulted in a remarkable alteration. However, glycerol and xylitol at both concentrations significantly increased hydration as compared to control and SLS-treated groups (Fig. 3).

SLS-induced inflammation led to a considerably faster local blood flow compared to the control animals. $10 \%$ glycerol and xylitol at both applied concentrations were found to be able to prevent the irritant-induced RBCV elevation (Fig. 4.(a)).

The number of sticking leukocytes detected in the control group was relatively low, while the SLS-treated group was characterized by significantly more leukocyte-endothelial interactions. $5 \%$ glycerol was able to partially decrease leukocyte sticking as compared to SLS-treated group. Xylitol, applied at both concentrations, as well as $10 \%$ glycerol treatment, effectively prevented leukocyte-endothelial interactions as compared to the control group (Fig. 4.(b)).

Epidermal thickness decreased by the exposure to SLS. Both polyols at all applied concentrations increased epidermal thickness considerably as compared to control and SLStreated groups (Fig. 5).

Addition of polyols to SLS prevented the elevation of lymphocyte number induced by the irritant (Fig. 6).

Exposure to SLS led to an elevation in the mRNA expression of IL-1 $\alpha$ but neither glycerol nor xylitol interfered with this alteration (data not shown). SLS-induced inflammation was 
accompanied by a considerable elevation in IL-1ß mRNA expression level as compared to the control group, which was inhibited only by the $10 \%$ glycerol treatment (Fig. 7.(a)).

mRNA expression of TNF- $\alpha$ was found to be significantly higher in SLS-treated group compared to control group. Again, 5\% glycerol had no effect, whereas $10 \%$ glycerol and xylitol applied in both concentrations were effective (Fig. 7.(b)).

Irritation with SLS also led to a considerable increase in MPO activity as compared to control group. Addition of the higher concentrations of the polyols to SLS effectively decreased the MPO activities (Fig. 8).

ATR-FTIR spectroscopy revealed the most characteristic bond of SLS assigned to $\mathrm{SO}_{2}$ stretch (asymmetric) at $1220 \mathrm{~cm}^{-1}$ (Fig. 9)[12]. It was found that both lower concentration polyol treatments yet modified the SLS penetration, however, significant decrease in the SLS amount in the deeper layers was observed applying the higher polyol treatments (Table 2). 


\section{Discussion}

SLS-induced inflammation is one of the most widely applied models of skin irritation[13], however, concentration of the experimentally administered SLS is a pivotal question[14]. According to previous data, 5\% of SLS is sufficient to evoke an inflammation with visible signs and to increase skin blood flow but does not induce epidermal necrosis[15]. In our experiment, 3-hour-exposure to 5\% SLS leads to an elevation of TEWL, which may be explained by hyperhydration of the stratum corneum (SC) and possible disorganization of lipid bilayers[16]. On the basis of previous studies, it has been expected that glycerol is able to moderate the irritant-induced TEWL changes[17, 18]. An explanation of the beneficial effects of glycerol may be that it stabilizes the lipids in SC and changes the water binding ability of proteins and lipids[19, 20]. However, increase in water holding capacity of SC reaches a plateau when applied glycerol concentration is higher than $3 \%$ after irritant challenge[18].

Our present study has shown that $10 \%$ glycerol and the xylitol treatment prevented the TEWL elevation caused by the 5\% SLS treatment. The reason why our findings differ from those of other studies may be because of differences in experimental setups. Atrux-Tallau et al. applied a considerably high concentration of SLS (10\%), which may have resulted in such severe SCdisruption that lipid-stabilizing effect of glycerol was not sufficient to sustain the barrier function[18]. However, according to our results, barrier damage induced by 5\% SLS could be repaired by $10 \%$ glycerol. Another important difference is that we applied the polyols together with SLS, while the mentioned other studies used the glycerol after the irritation.

In addition to glycerol, xylitol has been proven to be an effective anti-irritant when applied together with $0.1 \%$ of SLS[8]. In the present study, we provide evidence that xylitol can ameliorate a more serious inflammation, as well, and can exert its beneficial effect at a lower concentration compared to glycerol. Although it is difficult to predict the in vivo activity of 
polyols on the basis of their chemical structure[21], efficacy of xylitol may originate in its water binding capacity.

The results underline the moisturizer effect of both polyols in terms of other parameters, too. SC hydration was found to decrease considerably when the mouse skin was exposed to $10 \%$ of SLS[18], however, our results revealed that 5\% of SLS did not significantly reduce hydration in mice and both glycerol and xylitol raised hydration values. Moreover, the moisturizing effect of the polyols affected not only the SC, but also the viable epidermis, as it is demonstrated by elevation of epidermal thickness. Beyond skin hydration[22], inflammation-related edema[23] may also lead to a thicker epidermis. In our experiments, increased TEWL levels were detected, which may explain the reduction in epidermal thickness after SLS-treatment. According to our observations, the higher loss of water seems to exceed inflammation-caused edema and the thicker epidermis in polyol-treated groups shows the hydrating effect of glycerol and xylitol.

It is known that exposure to SLS is accompanied by increased dermal blood flow[15, 24]. SLS-induced elevation in RBCV was successfully moderated by $10 \%$ glycerol and both applied concentrations of xylitol. Glycerol may directly affect microcirculation: it has been reported that $100 \%$ anhydrous glycerol reduces blood flow velocity and vessel diameter of micro-vasculatures[25]. As glycerol we used was one magnitude lower than that in the mentioned study and application of polyols decreased not only dermal blood flow but also other indicators of inflammation, we presume that in this examination, decrease in RBCV in polyol-treated groups seems to originate in the anti-inflammatory activity of these compounds.

MPO is a frequently examined marker in murine models of ICD[26-29]. Our results suggest that SLS-treatment resulted in a considerable accumulation of neutrophil granulocytes, which 
was prevented by the application of polyols. Both IVM and MPO activity results confirmed that glycerol and xylitol can influence the neutrophilic activity in this model of irritation.

Although lymphocyte accumulation is more characteristic of atopic or allergic contact dermatitis, irritation may also be accompanied by elevated number of lymphocytes in the skin. According to the available literature data, application of SLS induces $\mathrm{T}$ cell-dominated inflammatory response in mouse oral mucosa[30]. In line with these results, our previous study has also demonstrated that exposure to SLS can lead to lymphocyte accumulation in the skin of SKH-1 mice[31]. This study provides evidence that the applied polyols are able to reduce the number of lymphocytes at the irritation site.

However, it is also necessary to characterize the impact of polyols on the cornerstone mediators of inflammatory response. Keratinocytes produce cytokines and chemokines (e.g. IL-1 $\alpha$, IL-1 13, TNF- $\alpha$, IL-8) after irritant challenge. The cytokines activate other cell types (e.g. dendritic, Langerhans, endothelial cells) whose cytokine release recruits neutrophil granulocytes, macrophages, mast cells and lymphocytes[32]. TNF- $\alpha$ is of special importance because migration of both dendritic and Langerhans cells require this cytokine and IL-1ß is also essential for the activation of the latter cell type[33, 34]. In murine models, elevation of TNF- $\alpha$ and IL-1ß can be expected in response to SLS treatment[35]. Furthermore, IL-1 $\alpha$ also contributes to the inflammatory response[36]. In this study, both glycerol and xylitol decreased the mRNA expression of IL- $1 \beta$ and TNF- $\alpha$, but had no effect on the IL- $1 \alpha$ levels. These may explain their beneficial effects on cellular factors of inflammation, but the exact mechanism via which glycerol and xylitol affect the cytokine expression requires further examinations.

Finally, as glycerol may reduce the average aqueous pore radius in the SC, hereby decreasing the penetration of irritants[37], one may presume that the anti-irritant and anti-inflammatory effects of glycerol and xylitol are just elucidated by the hampered penetration of SLS. 
Therefore, the penetration characteristic of SLS in the presence of the polyols, with combined tape stripping and ATR-FTIR spectroscopy[38, 39] was also studied. SLS penetration to the deeper SC layers decreased significantly at higher polyol concentrations, which is a novel feature for xylitol. However, this result is not completely in accordance with the previous observed effects of polyols, where xylitol merely proved to be effective already at lower concentration. In addition, hydrating effects of polyols is independent from their action on SLS penetration. Therefore, mechanism of action of glycerol and xylitol in acute ICD is thought to be partially due to their inhibition on SLS penetration but other unknown mechanisms should also exist.

In conclusion, glycerol and xylitol effectively maintain the barrier function of the skin in SLSinduced acute irritation and are able to hydrate the epidermis and exhibit anti-inflammatory activities. They prevent the inflammation-related increase in dermal blood flow, diminish the accumulation of neutrophil granulocytes and lymphocytes, moderate the expression of inflammatory cytokines and SLS penetration. The effective concentration of xylitol is lower than that of glycerol. According to these results both polyols may contribute to the prevention of ICD. 


\section{Acknowledgements}

The authors deeply thank the assistants for the excellent animal care. 


\section{References}

[1] Tan CH RS, Johnston GA. Contact dermatitis: Allergic and irritant. Clin Dermatol. 2014;32; 116-124.

[2] Andersen F HK, Petersen TK, Bindslev-Jensen C, Fullerton A, Andersen KE. Comparison of the effect of glycerol and triamcinolone acetonide on cumulative skin irritation in a randomized trial. J Am Acad Dermatol. 2007;56; 228-235.

[3] Rawlings AV CD, Dobrowski B. Moisturizer technology versus clinical performance. Dermatol Ther. 2004;17 49-56.

[4] Fluhr JW GM, Lehmann L, Lazzerini S, Distante F, Berardesca E. Glycerol accelerates recovery of barrier function in vivo. Acta Derm Venereol 1999;79; 418-421.

[5] Fluhr JW DR, Surber C. Glycerol and the skin: a holistic approach to its origin and functions. $\mathrm{Br}$ J Dermatol 2008;159; 23-34.

[6] Leite e Silva RV SM, Ferelli C,Gimenis JM, Ruas GW, Baby AR, Velasco MV, Taqueda ME, Kaneko TM. Hydrating effects of moisturizer active compound incorporated into hydrogels: in vivo assessment and comparison between devices. J Cosmet Dermatol. 2009;8; 32-39.

[7] Cohen S MY, Migron Y, Dikstein S, Shafran A. Water sorption, binding and solubility of polyols. J Chem Soc Faraday Trans. 1993;89; 3271-3275.

[8] Korponyai C KR, Erős G, Dikstein S, Kemény L. Antiirritant properties of polyols and amino acids. Dermatitis. 2011;22; 141-146.

[9] Szabó-Papp J SK, Oláh A, Szöllösi AG, Tóth BI, Czifra G, Bíró T. Differential effects of common moisturizer polyols on normal human epidermal keratinocytes. J Invest Dermatol. 2012;132; S58.

[10] Menger MD LM, Vollmar B. Viewing the microcirculation through the window: some twenty years experience with the hamster dorsal skin fold chamber. Eur Surg Res. 2002;34; 83-91.

[11] Kuebler WM AC, Schuerer L, Goetz AE. Measurement of neutrophil content in brain and lung tissue by a modified myeloperoxidase assay. Int J Microcirc Clin Exp 1996;16; 89-97.

[12] Bhise S.B. MS. Formulation and Evaluation of Novel FDCs of Antitubercular Drugs. J Pharm Research 2009;2; 437-444.

[13] Lee CH MH. The sodium lauryl sulfate model: an overview. Contact Dermatitis. 1995;33; 1-7.

[14] Agner T SJ. Sodium lauryl sulfate for irritant patch testing - a dose-response study using bioengineering methods for determination of skin irritation. J Invest Dermatol. 1990;95; 543-547.

[15] Jibry N MS. In vivo investigation, in mice and in man, into the irritation potential of novel amphiphilogels being studied as transdermal drug carriers. Eur J Pharm Biopharm. 2004;58; 107-119.

[16] Leveque JL dRJ, Saint-Leger D, Billy D. How does sodium lauryl sulfate alter the skin barrier function in man? A multiparametric approach. Skin Pharmacol 1993;6; 111-115.

[17] Andersen F HK, Petersen TK, Bindslev-Jensen C, Fullerton A, Andersen KE. Anti-irritants I: dose-response in acute irritation. Contact Dermatitis. 2006;55; 148-154.

[18] Atrux-Tallau N RC, Padois K, Denis A, Haftek M, Falson F, Pirot F, Maibach HI. Effects of glycerol on human skin damaged by acute sodium lauryl sulphate treatment. Arch Dermatol Res 2010;302; 435-441.

[19] Appa Y OD, Widjaja J, Asuncion A. Effect of glycerin on energy requirements and liquid crystallinity of model intracellular lipids. J Invest Dermatol. 1993;100; 587.

[20] Froebe C. Prevention of stratum corneum lipid phase transition in vitro by glycerol - an alternative mechanism for skin moisturization. J Soc Cosmet Chem 1990;41; 51-65.

[21] Sagiv AE MY. The connection between in vitro water uptake and in vivo skin moisturization. Skin Res Technol. 2003;9; 306-311.

[22] Benson HA KG, Edwards J, Liew YM, Wallace VP. Enhanced skin permeation and hydration by magnetic field array: preliminary in-vitro and in-vivo assessment. J Pharm Pharmacol 2010;62; 696701. 
[23] Yeom M KS, Lee B, Han JJ, Chung GH, Choi HD, Lee H, Hahm DH. Oral administration of glucosylceramide ameliorates inflammatory dry-skin condition in chronic oxazolone-induced irritant contact dermatitis in the mouse ear. J Dermatol Sci 2012;67; 101-110.

[24] Fullerton A RB, Serup J. Studies of cutaneous blood flow of normal forearm skin and irritated forearm skin based on high-resolution laser Doppler perfusion imaging. Skin Res Technol 2002;8; 3240.

[25] Zaman RT PA, Vargas G, Chen B, Dunn AK, Rylander HG 3rd, Welch AJ. Perfusion in hamster skin treated with glycerol. Lasers Surg Med 2009;41; 492-503.

[26] de Jongh C LR, Verberk MM, Kezic S. Differential cytokine expression in skin after single and repeated irritation by sodium lauryl sulphate. Exp Dermatol. 2007;16; 1032-1040.

[27] Kumar GK DR, Kulkarni NM, Mahat MYA, Gunasekaran J, Ashfaque M. Embelin reduces cutaneous TNF- $\alpha$ level and ameliorates skin edema in acute and chronic model of skin inflammation in mice. Eur J Pharmacol 2011;622; 63-69.

[28] Han MH YW, Lee H, Han SB, Lee K, Park SK, Yang KH, Kim HM, Kang JS. Topical application of silymarin reduces chemical-induced irritant contact dermatitis in BALB/C mice. Int Immunpharmacol 2007; 7; 1651-1658.

[29] De Vry CG VM, Lazarov M, Muhr E, Buelow R, Fong T, lyer S. Topical application of a novel immunmodulatory peptide, RDP58, reduces skin inflammation in the phorbol ester-induced dermatitis model. J Invest Dermatol 2005;125; 473-481.

[30] Ahlfors EE DJ, Lyberg T. The development of T cell-dominated inflammatory responses induced by sodium lauryl sulphate in mouse oral mucosa. Arch Oral Biol 2012;57; 796-804.

[31] Erős G KZ, Németh IB, Csizmazia E, Berkó S, Szabó-Révész P, Kemény L, Csányi E. The irritant effects of pharmaceutically applied surfactants. J Surfact Deterg 2014;17; 67-70.

[32] Gittler JK KJ, Guttman-Yassky E. Atopic dermatitis results in intrinsic barrier and immune abnormalities: Implications for contact dermatitis. J Allergy Clin Immunol. 2012;131; 300-313.

[33] Cumberbatch M KI. Dermal tumour necrosis factor-alpha induces dendritic cell migration to draining lymph nodes and possibly provides one stimulus for Langerhans cell migration. Immunology. $1992 ; 75 ; 257-263$.

[34] Cumberbatch M DR, Kimber I. Langerhans cells require signals from both tumour necrosis factor-alpha and interleukin-1 beta for migration. Immunology 1997;92; 388-395.

[35] Effendy I LH, Maibach HI. Epidermal cytokines in murine cutaneous irritant responses. J Appl Toxicol 2000;20; 335-341.

[36] Cumberbatch M DR, Groves RW, Antonopoulos C, Kimber I. Differential regulation of epidermal Langerhans cell migration by interleukins (IL)-1 $\alpha$ and IL-1ß during irritant- and allergeninduced cutaneous immune responses. Toxicol Appl Pharmacol. 2002;182; 126-135.

[37] Ghosh S BD. The role of sodium dodecyl sulfate (SDS) micelles in inducing skin barrier perturbation in the presence of glycerol. J Cosmet Sci. 2007;58; 109-133.

[38] Dias M NA, Guy RH, Hadgraft J, Lane ME. In vivo infrared spectroscopy studies of alkanol effects on human skin. Eur J Pharm Biopharm 2008;69; 1171-1175.

[39] Balázs B. FG, Berkesi O., Gyulai R., Berkó Sz., Budai-Szűcs M., Szabó-Révész P., Kemény L., Csányi E. Protein structure is changed in psoriatic skin on the unaffected region - Imaging possibility with ATR-FTIR spectroscopy. Microchem J. 2014;117; 183-186. 


\section{Figures}

Fig. 1: Experimental setup

Fig. 2: Transepidermal water loss (TEWL) after exposure to purified water (contr.) (white box), sodium lauryl sulfate (SLS) (black box), SLS plus glycerol (Gl) at 5\% (gray box) and $10 \%$ (checked gray box) or to SLS plus xylitol (Xy) at $8.26 \%$ (striped box) and $16.52 \%$ (checked white box). Values are given as percentage referred to TEWL before treatments. The plots demonstrate median with $25^{\text {th }}$ and $75^{\text {th }}$ percentiles. \#: $\mathrm{p}<0.05$ vs control, $\S: \mathrm{p}<0.05$ vs SLS-treated group.

Fig. 3: Changes in skin surface hydration in purified water-treated (white box), SLS-treated (black box), SLS+5\% glycerol-treated (gray box), SLS+8.26\% xylitol-treated (striped box), SLS+10\% glycerol-treated (checked gray box) and SLS+16.52\% xylitol-treated (checked white box) animals. Values are given as percentage referred to hydration before the treatments. The plots demonstrate median with $25^{\text {th }}$ and $75^{\text {th }}$ percentiles. \#: $\mathrm{p}<0.05$ vs control, $\S: \mathrm{p}<0.05$ vs SLS-treated group.

Fig. 4 4.(a): Red blood cell velocity (RBCV) in dermal capillaries after $3 \mathrm{~h}$ of treatment in groups 1-6. Values are given in $\mu \mathrm{m} / \mathrm{s}$, the plots demonstrate median with $25^{\text {th }}$ and $75^{\text {th }}$ percentiles. \#: p<0.05 vs control, §: p<0.05 vs SLS-treated group. 4.(b): Number of adherent leukocytes in the dermal postcapillary venules after $3 \mathrm{~h}$ of treatment. Cell numbers are referred to the area of vessel wall and given in $\left(\right.$ cell $\left./ \mathrm{mm}^{2}\right) * 10^{2}$. The plots demonstrate median with $25^{\text {th }}$ and $75^{\text {th }}$ percentiles. \#: $p<0.05$ vs control, $\S: p<0.05$ vs SLS-treated group.

Fig. 5: Photomicrographs of dorsal skin from groups 1-6 (hematoxylin-eosin staining). The graph shows the epidermal thickness in different groups $(\mu \mathrm{m}) .50 \mathrm{x}$ magnitude, scale bar represents $50 \mu \mathrm{m}$. Values are given as median with $25^{\text {th }}$ and $75^{\text {th }}$ percentiles. \#: $\mathrm{p}<0.05$ vs control, $\S: \mathrm{p}<0.05$ vs SLS-treated group. 
Fig. 6: Presence of lymphocytes in the treated skin in groups 1-6 (DAPI-staining, lymphocytes appear red). The graph demonstrates the number of CD3+ lymphocytes referred to 50 basal keratinocytes. 10x magnitude, scale bar represents $100 \mu \mathrm{m}$. Values are given as median with $25^{\text {th }}$ and $75^{\text {th }}$ percentiles. \#: $\mathrm{p}<0.05$ vs control, $\S: \mathrm{p}<0.05$ vs SLS-treated group.

Fig. 7 7.(a): Expression of IL-1ß mRNA after different treatments. Values are given as normalized relative expression. The plots demonstrate median values with $25^{\text {th }}$ and $75^{\text {th }}$ percentiles. \#: p<0.05 vs control, §: p<0.05 vs SLS-treated group. 7.(b): Expression of TNF- $\alpha$ mRNA in groups 1-6. Values are given as normalized relative expression. The plots demonstrate median values with $25^{\text {th }}$ and $75^{\text {th }}$ percentiles. \#: $\mathrm{p}<0.05$ vs control, $\S: \mathrm{p}<0.05$ vs SLS-treated group.

Fig. 8: Myeloperoxidase (MPO) activity in treated skin of different groups. Enzyme activity is referred to protein content and given as $\mathrm{mU} / \mathrm{mg}$ protein. The plots demonstrate median values with $25^{\text {th }}$ and $75^{\text {th }}$ percentiles. \#: $\mathrm{p}<0.05$ vs control, $\S: \mathrm{p}<0.05$ vs SLS-treated group.

Fig. 9: Attenuated total reflectance Fourier transform infrared spectroscopy (ATR-FTIR) spectra of the pure SLS, the 5\% solution of SLS and the non-treated stratum corneum. Absorbance is given in arbitrary units as a function of the wavenumber. 


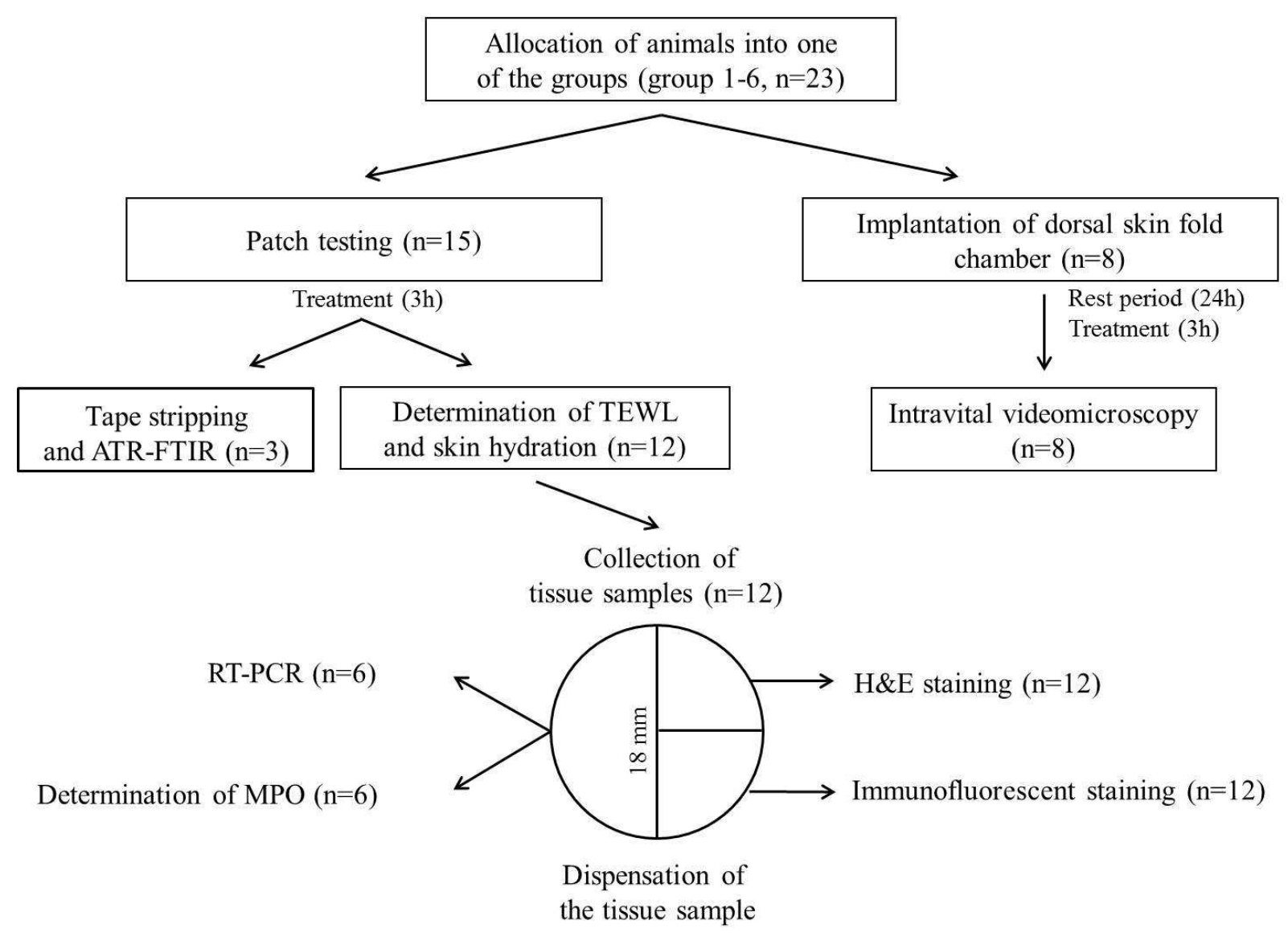




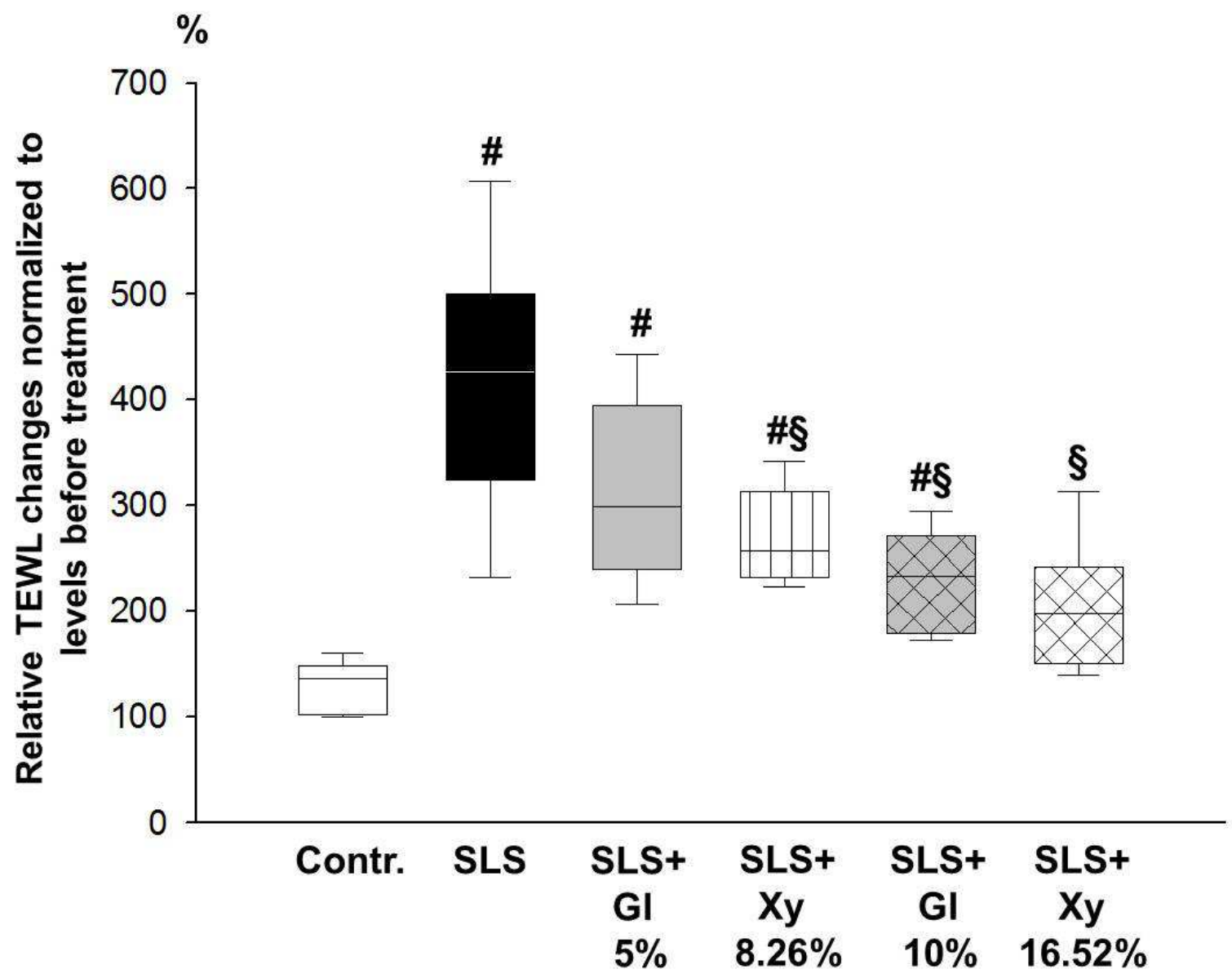




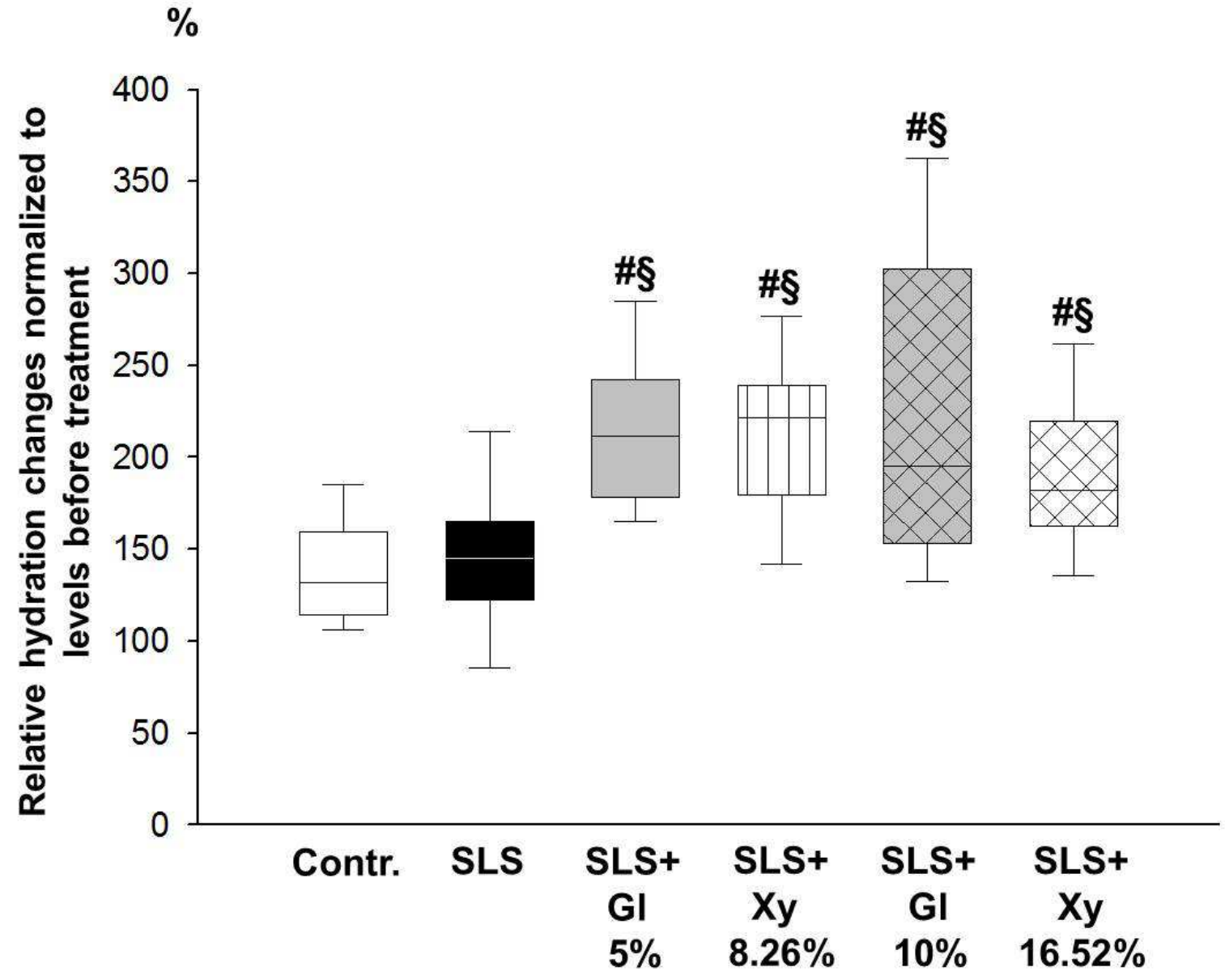


(a)

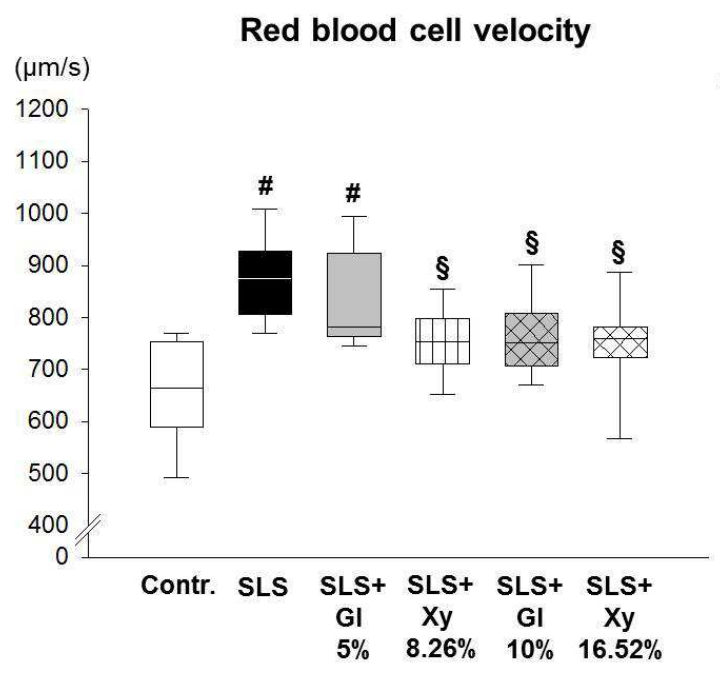

(b)

Relative number of adherent leukocytes $\left(\mathrm{cell} / \mathrm{mm}^{2}\right) \times 10^{2}$

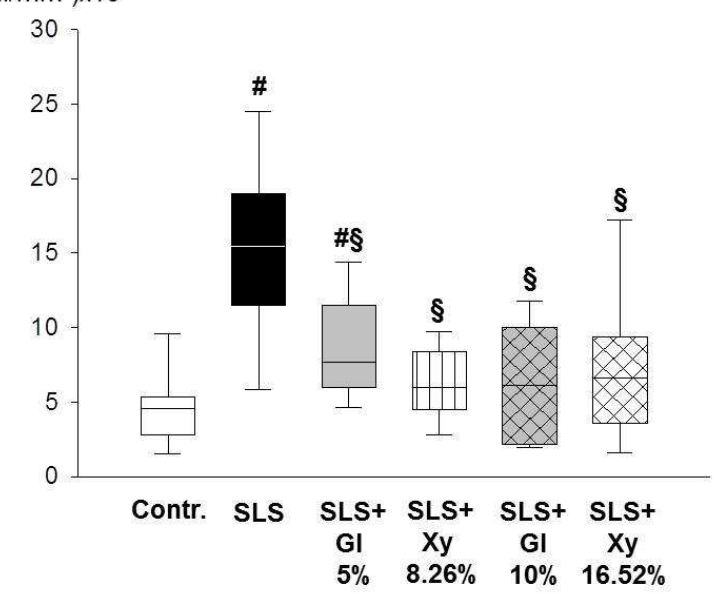



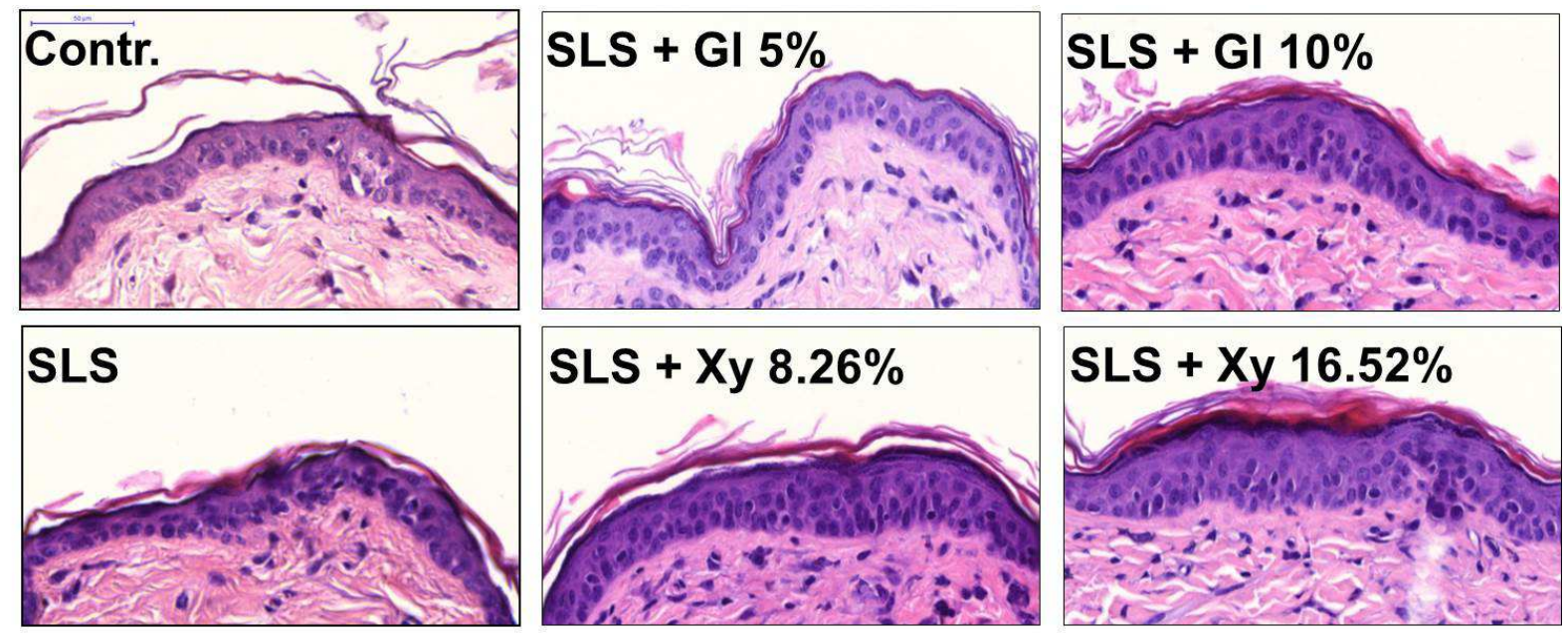

SLS + Xy 8.26\%

SLS + Xy 16.52\%

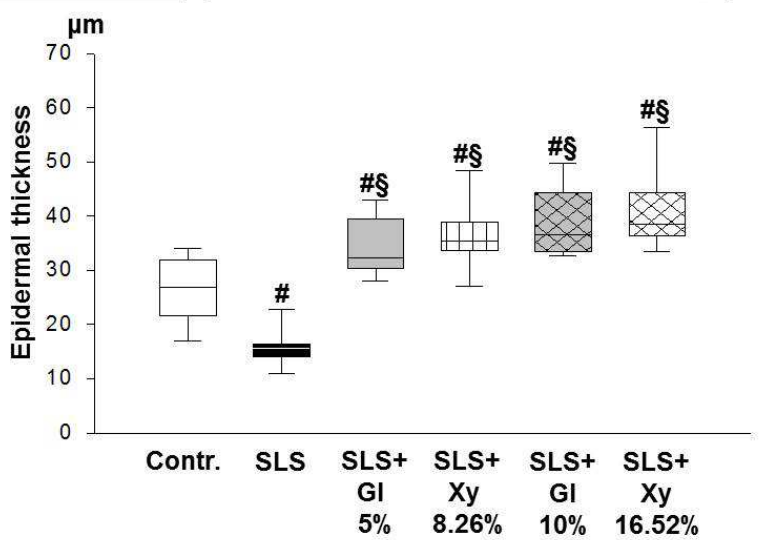




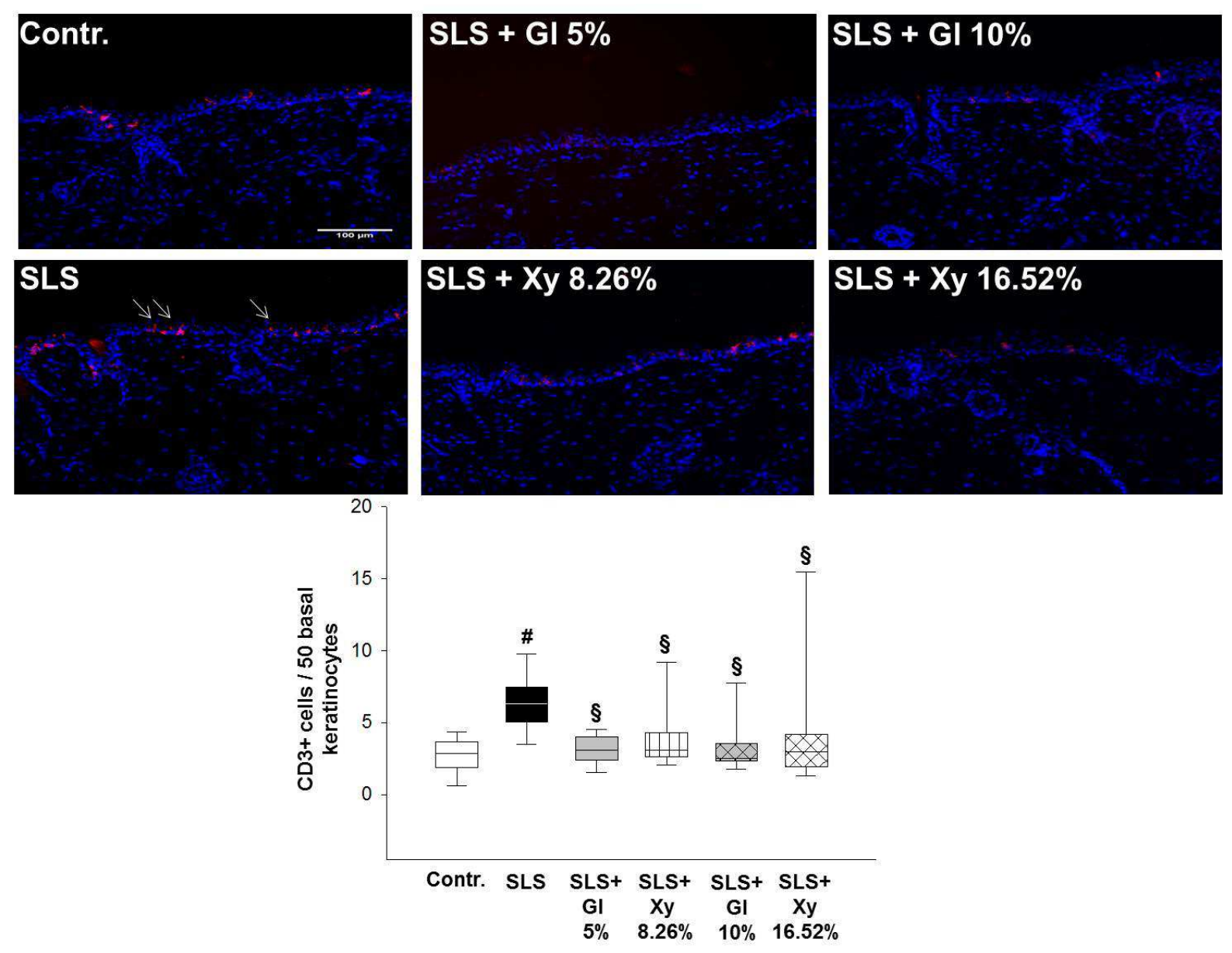


(a)

Norm. rel. expr.

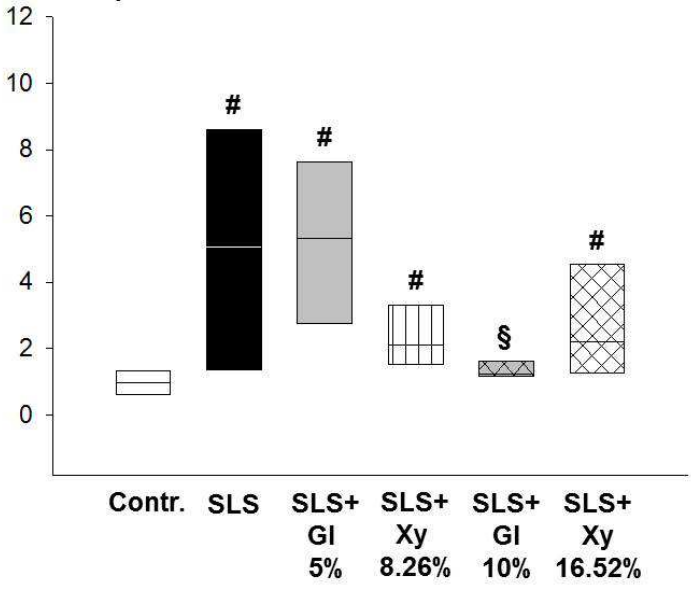

$I L-1 ß$ (b)

Norm. rel. expr.

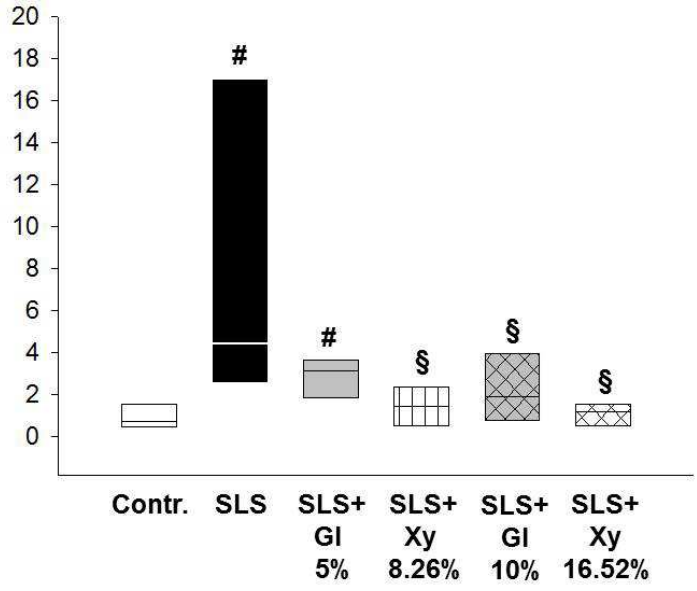

TNF- $\alpha$ 


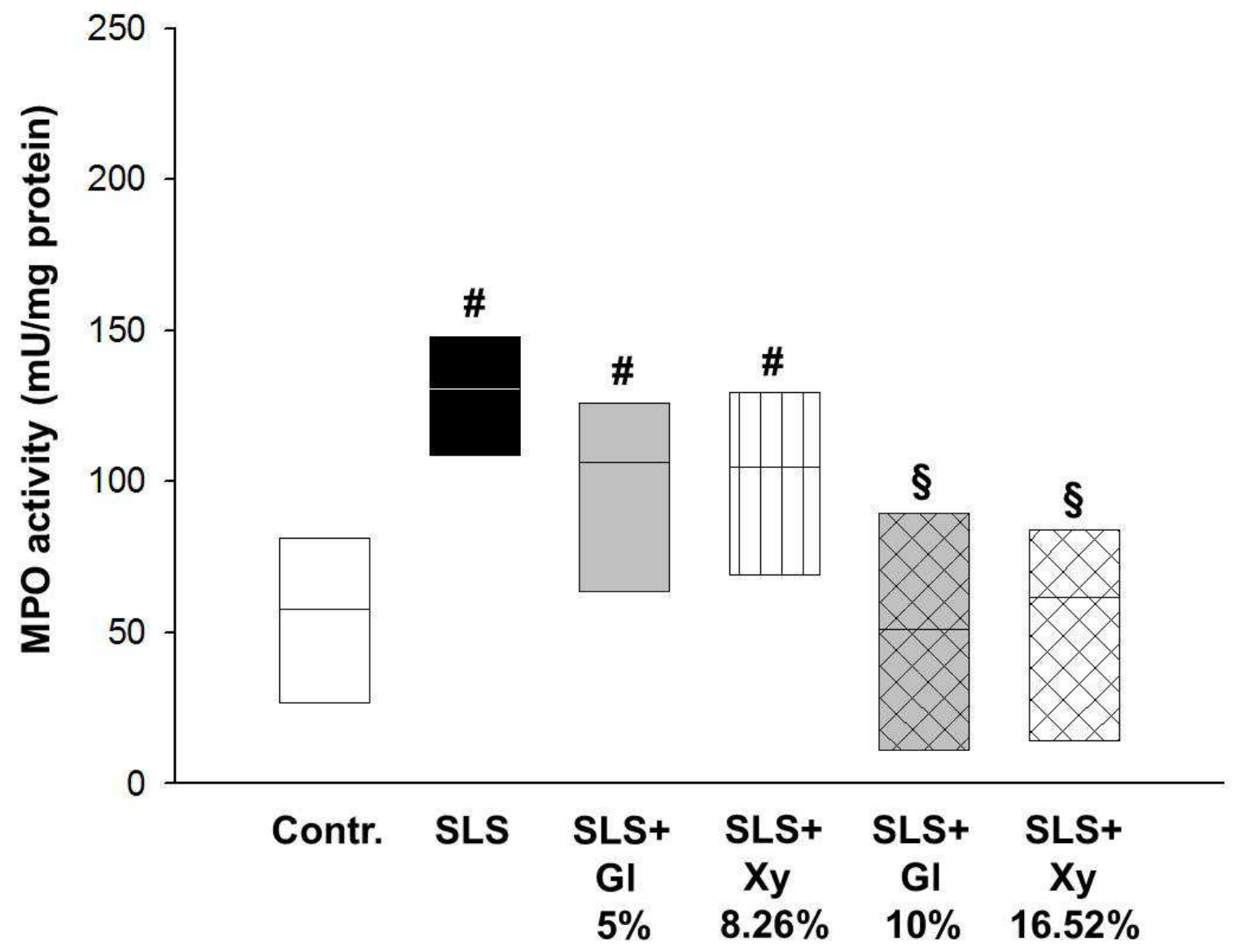




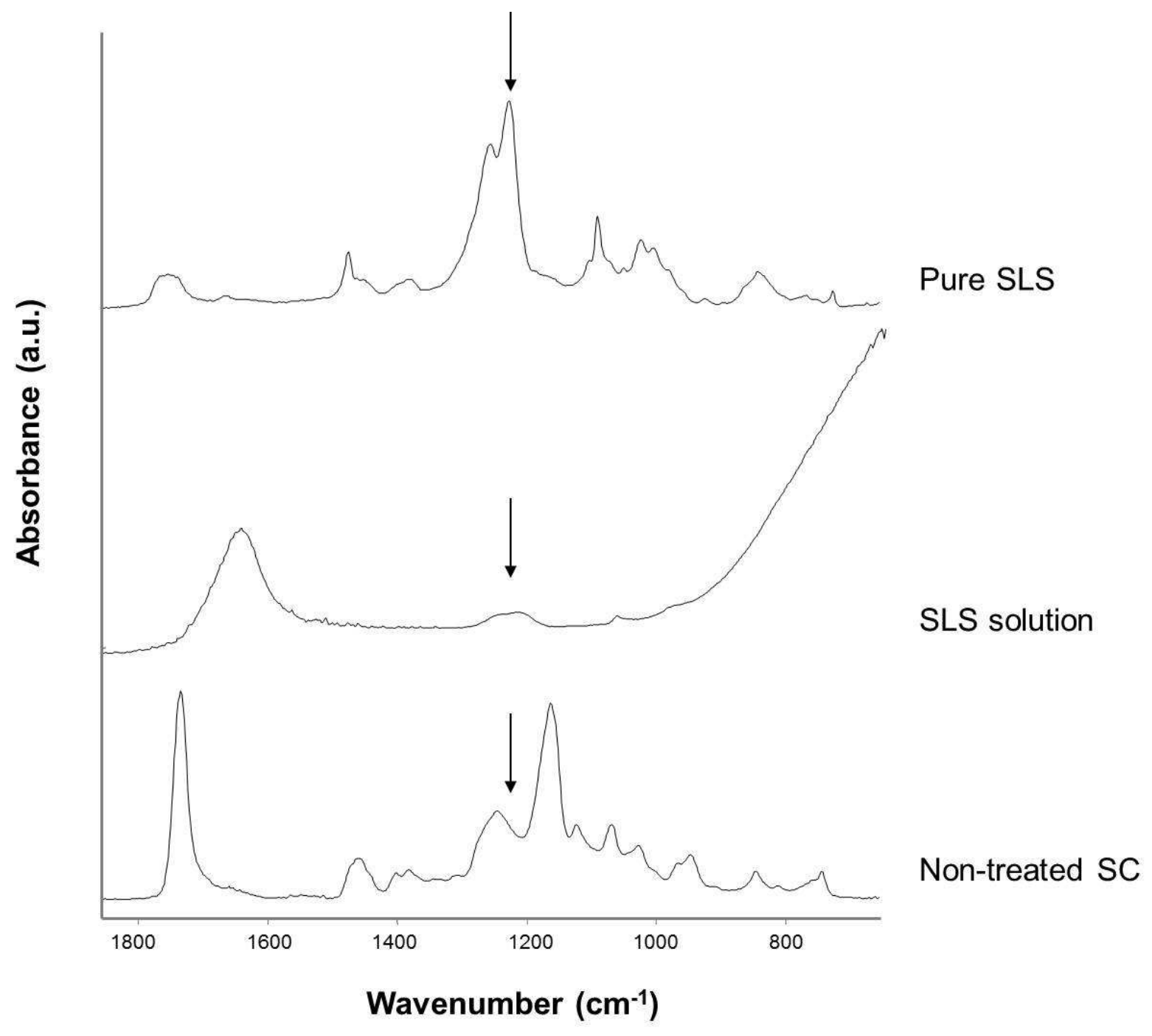


Tables

\begin{tabular}{|c|c|}
\hline Group & Applied agents and concentrations \\
\hline Group 1 & Purified water \\
\hline Group 2 & SLS (5\%) \\
\hline Group 3 & SLS (5\%)+ Glycerol (5\%) \\
\hline Group 4 & SLS (5\%)+ Xylitol (8.26\%) \\
\hline Group 5 & SLS (5\%) + Glycerol (10\%) \\
\hline Group 6 & SLS (5\%) + Xylitol (16.52\%) \\
\hline
\end{tabular}

Table 1: Study groups, applied agents and their concentration 


\begin{tabular}{|c|c|c|c|c|c|c|c|c|c|c|}
\hline & \multicolumn{2}{|c|}{$\begin{array}{l}\text { Group 2 } \\
(\text { SLS 5\%) }\end{array}$} & \multicolumn{2}{|c|}{$\begin{array}{c}\text { Group 3 } \\
(\mathrm{SLS}+\text { Glycerol 5\%) }\end{array}$} & \multicolumn{2}{|c|}{$\begin{array}{c}\text { Group 4 } \\
(\mathrm{SLS}+\text { Xylitol } 8.26 \%)\end{array}$} & \multicolumn{2}{|c|}{$\begin{array}{c}\text { Group 5 } \\
(\mathrm{SLS}+\text { Glycerol 10\% })\end{array}$} & \multicolumn{2}{|c|}{$\begin{array}{c}\text { Group 6 } \\
(\mathrm{SLS}+\text { Xylitol } 16.52 \%)\end{array}$} \\
\hline $\begin{array}{l}\text { Number } \\
\text { of layers }\end{array}$ & $\mathrm{M}$ & $\begin{array}{l}25 \mathrm{p} \\
75 \mathrm{p}\end{array}$ & $\mathrm{M}$ & $\begin{array}{l}25 \mathrm{p} \\
75 \mathrm{p}\end{array}$ & $\mathrm{M}$ & $\begin{array}{l}25 \mathrm{p} \\
75 \mathrm{p}\end{array}$ & $\mathrm{M}$ & $\begin{array}{l}25 \mathrm{p} \\
75 \mathrm{p}\end{array}$ & $\mathrm{M}$ & $\begin{array}{l}25 \mathrm{p} \\
75 \mathrm{p}\end{array}$ \\
\hline $1-4$ & 0.0226 & $\begin{array}{l}0.0137 \\
0.0264\end{array}$ & 0.0309 & $\begin{array}{c}0.0187 \\
0.0518\end{array}$ & 0.0318 & $\begin{array}{c}0.0181 \\
0.0468\end{array}$ & 0.0319 & $\begin{array}{l}0.0161 \\
0.0532\end{array}$ & 0.0196 & $\begin{array}{l}0.0134 \\
0.0360\end{array}$ \\
\hline $5-8$ & 0.0234 & $\begin{array}{l}0.0140 \\
0.0433\end{array}$ & 0.0223 & $\begin{array}{l}0.0122 \\
0.0300\end{array}$ & 0.0241 & $\begin{array}{l}0.0149 \\
0.0454\end{array}$ & $\begin{array}{c}0.00601 \\
*\end{array}$ & $\begin{array}{c}0.00143 \\
0.0134\end{array}$ & $\begin{array}{l}0.000 \\
*\end{array}$ & $\begin{array}{l}0.000 \\
0.0133\end{array}$ \\
\hline
\end{tabular}

Table 2 Intensities in the upper and lower layers of the treated stratum corneum (relative absorbance at $1220 \mathrm{~cm}^{-1}$ SLS)

Median values (M) with $25^{\text {th }}$ and $75^{\text {th }}$ percentiles (25p and 75p) of relative absorbance corresponding to the amount of SLS penetrated to the stratum corneum layers (1-4: upper layers, 5-8: lower layers) in the five groups. Group 2 was used as a positive control. * $\mathrm{p}<0.05$ vs. SLS-treated group. 